\title{
Oil fossilization of silicified microbes in black chert from the 340 Ma Red Dog Zn-Pb deposit, Alaska: Implications for ancient microfossils
}

\author{
BIRGER RASMUSSEN1 AND JANET R. MUHLING1
}

${ }_{1}$ School of Earth Sciences, The University of Western

Australia, Perth, Western Australia 6009, Australia.

Microfossils are an important source of information about the antiquity of life and the nature of the early biosphere. However, the preservation of microbes in the rock record is a rare event. In order to interpret the information they contain it is important to understand how they formed. The preservation of carbonaceous microfossils in Precambrian black cherts has generally been attributed to early diagenetic silica precipitation, which seals and protects the original cellular carbon. However, while the silicification of bacteria can preserve cellular morphology, organic molecules are rapidly destroyed after death in modern hot springs, raising questions about the origin of carbonaceous matter in ancient microfossils in Precambrian black cherts.

We have examined the origin of organic carbon in filamentous microfossils in black chert pebbles from the 340 million-year-old Red Dog $\mathrm{Zn}-\mathrm{Pb}$ deposit, Alaska. Our observations show that black carbonaceous microfossils are associated with hairline fractures stained brown by solidified oil. Transmission electron microscopy of thin foils cut across the black filaments, reveals the presence of thin films of carbonaceous matter ( $<50 \mathrm{~nm}$ thick) lining nano-quartz crystals in intracellular cavities. The transition from carbonrich black filaments around oil-stained fractures to barely visible filaments in background white chert indicates that most, if not all, of the indigenous carbon was destroyed.

Our observations suggest a scenario whereby silica nucleated on bacterial cell walls, followed by the decomposition of cellular carbon. The resultant silica-encased moulds were subsequently infiltrated by oil. The possibility that this process affected older carbonaceous microfossils awaits further investigation. However, hydrocarbons are likely to have been formed since the early Precambrian, generated either by abiogenic processes (e.g. FischerTropsch-type synthesis) or thermal maturation of organic-rich sediments. Hence, hydrocarbons were almost certainly present at Earth's surface when ancient microbes were being silicified in sediments up to 3.5 billion years ago. Indeed, there is abundant evidence for the migration of hydrocarbons in Archean cherts. This prompts potential questions about the preservation of Earth's oldest microfossils-is the intracellular carbon indigenous, and if not, does that preclude a biological origin? 